\title{
La familia como respuesta a las demandas de individualización: ambivalencias $\mathrm{y}$ contradicciones
}

\author{
Andreu López Blasco
}

Association of Regional and European Analysis (AREA)

andreublasco@terra.es

\section{Resumen}

Para evaluar los temas relacionados con la llamada «emancipación» de la juventud, queremos presentar la comparación entre la situación y las vivencias de los padres (antes), la evolución que ha tenido lugar y las estrategias, perspectivas y vivencias de la juventud actual.

¿Qué significados tenían «antes» las relaciones en la familia?: contar con un modelo más o menos estable de funcionamiento, de comportamiento, de formación y de aprendizaje de una profesión, de metas de formación, de fases de formación hasta llegar a la jubilación. Modelos de comportamiento y modelos de significación de la vida, tipos de unión existentes entre los grupos primarios, ligaduras ideológicas cerradas (partidos, Iglesia), transición a formas de vida estable (matrimonio), movilidad reducida (pocos cambios en las cualificaciones o movilidad local).

«Hoy» las dependencias son cambiantes y diferenciadas como consecuencia de la inseguridad y de la incerteza en el futuro, en las formas de vida y en los comportamientos Para poder asegurar su existencia y crear nuevas formas de vida o adaptarse a los diferentes estándares, los miembros de las familias permanecen juntos mucho más tiempo (la familia como hotel, como soporte), como respuesta a las demandas de la individualización, como una forma de amortiguar los riesgos y/o asegurarse ante los mismos.

Se pretende hacer una aportación al análisis de las ambivalencias en la que viven las madres y los padres que, entre 1970 y 1989, tenían la misma edad de los jóvenes y las jóvenes que, entre 1994 y 2004, tienen entre 20 y 25 años.

Palabras clave: jóvenes, transiciones, segunda modernidad, individualización, capital social, padres y transiciones, la familia como red de apoyo, función de la familia, grounded theory, investigación cualitativa.

\section{Abstract. Family as the answer to demands of individualisation: ambivalences and contradictions}

To evaluate the topics related to the so-called «emancipation» of youth, we would like to present a comparison between the situation and the experiences of parents, the evolution that has been taking place and the strategies, views and experiences of young people.

¿What kind of meanings had familiar relations in "the past»?: to have a more or less stable model, that regulated behaviour, training and learning, the training goals and the training steps until retirement. Behaviour models and life-meaning models, existing types of unions between primary groups, closed ideological bonds (political parties, the Church), 
transition towards more stable forms of living (marriage), limited mobility (little change in qualifications or local mobility).

"Today» dependencies are changing and differentiating as a consequence of insecurity and uncertainty about the future, the forms of living and the behaviours. To secure their existence, create new ways of living together or adapt to new standards, family members remain together much more time (family as a hotel, as a holder), because of demands of individualisation, as a way to absorb risks and/or hide from them.

We are trying to make a contribution to the analysis of the ambivalences in which parents live. Parents that between 1970 and 1989 were the same age that young people are now, that is, between 20 and 25 years old.

Key words: youth, transitions, late modernity, individualisation, social capital, parentstransitions, family as network, family as function, grounded theory, qualitative research.

\section{Sumario}

Introducción

1. Fuentes empíricas del artículo

2. Ambivalencias entre la «biografía normal» de los padres y la «biografía bricolaje» que tienen que crear los hijos/as
3. La creación de un hogar dentro del hogar de los padres: una forma de «salir de casa de los padres"

4. Interpretaciones

Bibliografía

\section{Introducción}

En una publicación de la Diputación de Barcelona (2002), Estudi d'orientació de les polítiques locals de joventut a la provincia de Barcelona, en el apartado sobre «Principios orientadores de las políticas de juventud», se pueden leer las afirmaciones siguientes de un joven trabajador entre 16 y 18 años:

Sí que haurien de fer coses, perquè no es preocupen gaire. Tenen com un projecte que diuen «la gent jove després creixerá i serà com els adults normals», i no sé com sortirà això. Em sembla que no es preocupen gaire [...] Em sembla que ens veuen com si tot el que fem, totes les afeccions que podem tenir siguin passatgeres, que després les deixes i et preocupes només per la feina i per tenir la teva família i ja està. I no crec que [...] potser en alguns passarà que deixaran les afeccions $\mathrm{i}$ es tornaran més centrats $\mathrm{i}$ tot això, però hi haurà gent a qui agradarà seguir amb la seva vida i seguir amb les seves coses encara que treballi i hagi de mantenir una família. O potser alguns no tindran família. (p. 54)

Este joven entre 16 y 18 años expresa acertadamente lo que hemos denominado «desestandarización de las transiciones» (Du Bois-Reymond, López Blasco, 2003). En el modelo de la era industrial, la juventud ha sido conceptualizada como una fase de la vida, una moratoria que sigue a la infancia y sirve como preparación a la edad adulta. La transición de la infancia a la madu- 
rez se consideraba como un proceso lineal en el que se generaban biografías normalizadas y diferentes según el sexo, estructuradas por el empleo remunerado en el caso de los hombres y por el rol de madres y amas de casa en el de las mujeres. Sin embargo, en los últimos años, se observa una brusca ruptura de esta linealidad de las biografías que implicaban una llegada "natural» a la vida adulta después de la infancia y la juventud, la cual se concibe como una condición vital caracterizada por su reversibilidad, incertidumbre y vulnerabilidad.

En la discusión teórica, se ha dejado de hablar de la juventud y la vida juvenil como un grupo de edad, cohorte o generación y nos centramos más en el estudio de la transición del joven a la vida adulta como una fase de vida organizada socialmente, en la que influyen las condiciones y las ofertas estructurales como educación, trabajo, sanidad, bienestar, vivienda, etc., que están, además, reguladas dentro de un marco jurídico y político, con la particularidad de que el uso que los jóvenes hacen de estas condiciones es al mismo tiempo individual y como miembro del grupo social.

En la sociedad actual, que definimos como postmoderna o de la segunda modernidad, los jóvenes toman decisiones y asumen sus responsabilidades en aspectos de la vida como son el matrimonio, la tenencia de hijos o las relaciones, y lo hacen de un modo individual y subjetivo, no impuesto ni determinado por otros, por la tradición o las costumbres. Los jóvenes no están simplemente determinados por la sociedad, ni son meros «objetos de socialización». Son actores reales que manejan o al menos influyen sobre las condiciones de sus vidas.

La sociedad de la segunda modernidad se caracteriza por la individualización, concepto sociológico que describe una sociedad que ha perdido las anclas tradicionales, las normas valederas para todos, que ha superado pautas de comportamiento unidas a roles predeterminados. El rol-making de los interaccionistas encuentra en la postmodernidad su máxima expresión, en cuanto que cada uno debe crear su propio rol, como hombre, como mujer, como hijo o hija, como persona consumidora, etc. Unido a ello va el concepto de riesgo, de incertidumbre, de soledad, de autorresponsabilidad. No son los estándares sociales los que imperan, sino los individuales, los propios, con ello se abre la puerta a una pluralidad sin fin (Hernández y López Blasco, 2005).

Podríamos enumerar tres condicionantes que influyen en nuestra vida en la sociedad actual: primero, nuestro actuar está marcado por la inseguridad; segundo, existe un riesgo constante y aunque intentamos calcular y valorar el alcance de nuestras decisiones, siempre hay sorpresas y nos podemos equivocar; tercero, nuestro comportamiento y nuestras actuaciones están influenciadas por los cambios que se dan en la confianza que nos merezcan los estilos y estándares de vida vigentes hoy y mañana ya anticuados: opiniones, juicios, valoraciones que hoy nos dan confianza y mañana están obsoletos (Bauman, 2004). Ante una visión de múltiples oportunidades, los jóvenes de hoy están relegados a sí mismos, forjan sus propias biografías que aparecen como biografías de bricolaje, diseñan sus propios itinerarios biográficos, mayoritaria- 
mente sin «una hoja de ruta» elaborada con anterioridad por la sociedad y sus instituciones (familia, escuela, formación, empleo).

Una de las discrepancias más profundas que se dan entre la modernidad y la postmodernidad es la ambivalencia: las personas en nuestros ámbitos culturales postmodernos tienen que ver en su vida cotidiana con actitudes, comportamientos y relaciones cotidianas que aparecen como contradictorias, y que pueden serlo, pero que pueden no excluirse, como señala Bauman, ya que resultan una característica de nuestra sociedad. Lo importante ya no es la disyunción, hay que decidirse por esto $o$ aquello (entwederloder), sino la copulación, las personas pueden tomar decisiones diferentes y contradictorias (hacer esto $y$ aquello (undlund). Todo puede valer en una sociedad actual en la que sólo está determinado el punto de partida pero no el de llegada (Bauman, 1996, p.73119). Los jóvenes y las jóvenes pretenden conseguir al mismo tiempo una integridad biográfica y, para ello, buscan también modelos de comportamientos que les den seguridad y claridad, buscándolos en los ambientes subculturales, en el grupo de iguales y, por otra, buscan modelos sociales para desarrollar su biografía, y esta aparente contradicción es lo que se expresa en términos de ambivalencia.

La nueva situación a la que se enfrentan los jóvenes en la sociedad postmoderna genera una dinámica distinta y nueva: los jóvenes y las jóvenes permanecen más años en la familia de origen, y siguen estrategias de retardamiento de los procesos de adultez, en contraste a como lo hicieron sus padres, pero, al hacerlo simultáneamente, generan innovaciones en los procesos de transición y en las estructuras sociales, como ejemplos encontraríamos las nuevas formas de relacionarse entre sí, las relaciones de pareja, las formas de relacionarse en la familia de origen, y la paternidad y maternidad. Con sus nuevas formas de relación, no sólo rompen un eslabón en la cadena de la transición hacia la vida adulta, sino que también cuestionan al mismo tiempo la función y las dimensiones de la familia de origen. Un potencial conflictivo que afecta a las mismas bases de la sociedad. Ellos y ellas, relegados a sí mismos y desde el parapeto todavía vigente y válido de la familia de origen, crean sus espacios distintos y diferenciados en los que, libres de las estructuras sociales (mercado de trabajo, matrimonio, procreación), crean sus redes sociales, de relaciones e intercambio, pero al hacerlo quedan cuestionadas las instituciones reproductoras de la propia sociedad.

En los últimos años, se han llevado a cabo investigaciones sobre las condiciones de vida de los jóvenes y las jóvenes entre 15 y 29 años. Si se investigan las condiciones que han llevado a los cambios en las formas de desarrollar y estructurar su vida, sería de una importancia determinante analizar y aclarar el contexto social de este grupo de edad. El grupo de edad entre 20 y 25 años marcaría más o menos la media entre los 15 y los 29 años. La mayoría de los jóvenes de 19 años todavía están estudiando, están aprendiendo un oficio o ya han tenido una experiencia laboral y las primeras experiencias de la precariedad del mercado de trabajo. Otra parte de jóvenes, a los 24-25 años todavía no ha terminado los estudios, todavía no ha tenido un empleo y todavía no vive con su pareja, ni vive en un hogar propio. 
Para descubrir el punto de referencia en el que se orientan las madres y los padres de los que hoy tienen entre 15 y 29 años sería importante tener en cuenta el momento de la sociedad en que vivían cuando ellas y ellos tenían entre 20 y 25 años. Podríamos fijar como espacio temporal los años que van desde 1970 (madres de los que hoy tienen 29 años tenían en 1970 esa misma edad) y 1989 (las madres de los que hoy tienen 15 años). Las madres que entre 1970 y 1989 tenían entre 20 y 25 años, podríamos relacionarlas con los jóvenes que entre 1994 y 2004 tenían entre 20 y 25 años. Partiendo de esta referencia temporal, podríamos preguntarnos qué acontecimientos acaecidos en los últimos 35 años (1970-2005) han acuñado las condiciones de vida de las personas y cómo han podido influir en las relaciones familiares y en las formas de comunicarse en el espacio en el que conviven los padres y los hijos ${ }^{1}$.

Creemos que lo importante no es determinar el mayor o menor porcentaje de jóvenes que han abandonado la familia de origen, sino más bien analizar las decisiones que los jóvenes y las jóvenes van tomando, sus características y condicionantes, así como los procesos que les llevan a decidirse por una $\mathrm{u}$ otra forma de vida y las nuevas funciones que tienen que adoptar las familias.

Abandonar la casa de los padres o alargar la permanencia en la misma es el resultado de un largo proceso, que en algunos casos puede ser incluso reversible y que responde al proceso de individualización, en el que los jóvenes tienen que tomar decisiones y son ellos mismos los responsables del éxito o el fracaso de las mismas.

Los resultados de la investigación que hemos llevado a cabo en España, en el marco del proyecto FATE, nos llevan a la conclusión de que no se puede valorar si la decisión de abandonar o de quedarse en casa de los padres es normal o correcta, esto es: alargar la estancia en casa de los padres no es lo «incorrecto» y abandonarla tempranamente sería «lo normal», fenómeno que además se suele denominar positivamente como "emancipación». Hay que tener en cuenta muchos factores y condicionantes para valorar los deseos de autonomía o independencia de los jóvenes y las jóvenes.

En el Informe Juventud en España 2004 (2005), hemos podido fundamentar empíricamente (capítulo 2: «Familia y transiciones: individualización y pluralización de formas de vida») que abandonar o permanecer en la familia de origen es una decisión voluntaria de los jóvenes y las jóvenes y que

1. En los estudios empíricos que conocemos sobre "familia y jóvenes», no se suele tener en cuenta el trasfondo de los grandes cambios que se han dado en la sociedad y sus posibles efectos sobre las relaciones que tienen lugar en el ámbito del hogar. Habría que estudiar la configuración de la vida familiar bajo las condiciones mutables de la sociedad de la segunda modernidad.

Entre los contrastes de la sociedad de los años 1970-1980 y el mundo actual, podríamos señalar la necesidad de conquistar la libertad, la democracia y el disfrute de la misma. La comparación tendría que hacer referencia, entre otros, a los cambios en el modelo y la función de la familia, las libertades, la sexualidad, el tener que vivir con el paro, el incremento del Estado de bienestar y los grandes cambios vividos al final del franquismo, la transición y la caída del muro de Berlín y la generalización de la globalización. 
forma parte de estrategias concretas. Ante la necesidad de tomar decisiones, los jóvenes y las jóvenes optan por esperar a tiempos mejores; de manera que, siempre que la situación socioeconómica de las familias lo permita, aprovechan la estancia para aumentar su capital social, para ahorrar, para preparar su independencia, para mejorar sus opciones de éxito. Mientras esperan, utilizan los servicios del hogar y las relaciones personales de la familia. También se constata que una mayoría de los jóvenes y las jóvenes que abandonan el hogar en edades tempranas solamente tienen estudios primarios y sus padres suelen desarrollar trabajos para los que no se necesita una determinada cualificación.

Lo importante es descubrir cómo intentamos solucionar todos nosotros la difícil tarea de encontrar «soluciones biográficas a las contradicciones sistémicas» en las que tenemos que vivir (Beck). Una tarea casi imposible de resolver y a cuya solución estamos, por otra parte, obligados.

\section{Fuentes empíricas del artículo}

Durante tres años (de octubre de 2001 a diciembre de 2004), diez equipos de investigación en nueve regiones de la Unión Europea (Alemania del Este, Alemania del Oeste, Bulgaria, Dinamarca, España, Italia, Países Bajos, Portugal y Reino Unido) hemos analizado diversos aspectos de las vidas de los jóvenes realizando una investigación cualitativa con jóvenes y sus padres y una investigación a través de un cuestionario estandarizado que hemos pasado a jóvenes en cada una de las nueve regiones, según diferentes niveles de educación y formación (han contestado unos 2.000). Los temas de esta investigación incluían las transiciones de la escuela al trabajo, las transiciones de los jóvenes desde la familia de origen, el apoyo de las familias, las culturas familiares y las representaciones acerca de los planes de vida de lo que significa alcanzar la adultez. El trasfondo del proyecto era el análisis comparado de las transiciones en las nueve regiones.

La investigación cualitativa se basa en 72 entrevistas, 42 de la cuales han sido realizadas a jóvenes y 30 , a sus padres. Las personas entrevistadas viven en ámbitos urbanos o semiurbanos, ubicados en Valencia y su área metropolitana y en Pamplona y su comarca. La elección de la muestra se basaba en el nivel educativo y en el sexo: 14 entrevistas por nivel educativo, ISCED 0-2, ISCED 3 y ISCED 4, de las cuales 7 entrevistas eran por sexo en cada uno de los niveles educativos. El acceso a los entrevistados se llevó a cabo de forma aleatoria sobre el listado de los jóvenes y las jóvenes que habían contestado al cuestionario (primera fase del proyecto de investigación); a los padres y madres accedimos a través de sus hijos e hijas (sólo conseguimos entrevistar a 30 padres y madres de los 42 jóvenes entrevistados). Todas las entrevistas se realizaron teniendo como base un guión —-siguiendo las recomendaciones metodológicas-, fueron grabadas, transcritas y se han elaborado todo tipo de protocolos y resúmenes: sobre el lugar en el que se realizó la entrevista, sobre las actitudes, las reacciones ante algunas preguntas, sobre todos aquellos detalles que 
nos dieran alguna información sobre el entorno social y económico de las familias. Las entrevistas tienen una duración entre 45 y 60 minutos.

Fundamentamos la evaluación cualitativa en el paradigma de la grounded theory (Corbin y Strauss, 1990), que nos ayuda a interpretar los datos usando procedimientos inductivos. La grounded theory es algo inductivamente deducido del estudio de un fenómeno analizado. Esto es, consiste en descubrir, desarrollar y, provisionalmente, averiguar, mediante el análisis sistemático y las relaciones que tienen entre sí las experiencias, expresiones, datos recogidos durante la entrevista, durante la observación. La recogida de datos, los análisis y la teoría se encuentran en relación recíproca de unos con otros.

En la evaluación cualitativa que hemos llevado a cabo, no hemos empezado con una teoría y después hemos visto si la podemos probar, sino que ha sido el análisis y los diferentes pasos de la codificación la que nos ha llevado a formular algunas conclusiones. La base del enfoque grounded theory se basa en la codificación. El procedimiento de codificar implica aquellas operaciones en las que los datos son descompuestos, conceptualizados y juntados de nuevo de forma diferente a la que inicialmente han sido obtenidos; en la codificación reside el concepto analítico de la grounded theory.

Aplicando todos los pasos que propone la grounded theory, llegamos a descubrir una serie de indicadores de fenómenos, que son generalizables para las circunstancias o las situaciones de las familias y los jóvenes que hemos investigado.

Los resultados de la investigación abren nuevas perspectivas sobre la nueva función de la familia, las políticas europeas relacionadas con la familia, el aprendizaje de por vida, el empleo y la inclusión social (Families and Transitions in Europe (2001-2004): A comparative analysis in nine European regions (5० programa marco), http://www.socsci.ulster.ac.uk/policy/fate/fate.html).

El acercamiento científico que hemos realizado en el Informe Familias y Transiciones en España hemos tenido en cuenta una imagen abierta de la familia, sin utilizar una definición previa, sin hacer referencia a las diferentes formas que históricamente han ido teniendo las familias, queríamos saber cómo son y cómo responden en diferentes espacios y ámbitos temporales a los cambios sociales. La metodología utilizada nos ha facilitado constatar una vez más que las familias se desarrollan entre tradiciones y respondiendo a las nuevas condiciones de la sociedad postmoderna. Esta doble lógica significa que las familias reproducen formas de vida tradicionales y desarrollan nuevas formas; en unos casos, pueden estar más influenciadas por la tradición y, en otros, por las nuevas condiciones de vida, un reflejo de las ambivalencias y contradicciones de la sociedad postmoderna (Bauman, 1996).

La evaluación de las entrevistas aporta muchos ejemplos de cómo las familias reproducen anteriores formas de vida familiar y al mismo tiempo desarrollan nuevas formas de vida más acordes con las demandas de la sociedad postmoderna. Las familias desarrollan formas de vida que están más influenciadas por la tradición y, al mismo tiempo, otras formas de vida más acordes con la sociedad actual, una forma de adaptarse a la vida postmoderna. La codifica- 
ción de las entrevistas muestran una «activa» convivencia de diferentes modelos de familia según sean las condiciones de vida y los momentos biográficos de las mismas.

En algunos momentos de su biografía predomina una orientación más tradicional, centrada en la producción, que quiere que sus hijos estén más cualificados que ellos mismos, que puedan responder a las demandas de la sociedad, asumiendo para ello la función de socializar a los hijos. Contemplan la socialización como un proceso de aprendizaje, donde niños y jóvenes aprenden a comportarse según las normas y las reglas de la sociedad y se sienten responsables del desarrollo de los niños y los jóvenes.

Junto a esta función productiva y de trasmisión de normas y roles, aparece una nueva función, que podemos denominar «de apoyo al proceso de individualización de sus hijos e hijas», aunque este apoyo a sus hijos esté, en muchos casos, en contradicción con sus propios deseos o con sus expectativas de futuro. Apoyan los planes de futuro de sus hijos y al mismo tiempo esperan que éstos reproduzcan sus estilos de vida, sus costumbres. Los padres, sin estar muy convencidos de lo que hacen, se convierten en lo que denominamos «una red de apoyo» (Biggart, Walter y otros, 2004).

Compaginan actitudes que podemos denominar de "post-modernidad» (como libertad, autonomía versus individualización, coparticipación en la familia) y, al mismo tiempo, comportamientos de premodernidad (solidaridad obligada, familia como unidad con prestación de servicios recíprocos, comunidad de bienes). Ofrecen todos los apoyos posibles, se sacrifican para que sus hijos puedan adquirir más conocimientos educativos de los que ellos alcanzaron, esperan que ellos puedan «subir» socialmente, pero al mismo tiempo sueñan con que sus hijos repitan su biografía relacional y que se queden a vivir en su pueblo o, todo lo más, en el de al lado. Quieren tener a sus hijos cerca, aunque también temen, porque ya conocen algunos casos de sus amigos, que sus hijos encuentren trabajo en otros países. Suelen estar tan preocupados por el posible ascenso social de sus hijos a través de los estudios, que aceptan todas sus condiciones. Los padres se acomodan a los deseos de sus hijos.

La metodología utilizada también pone de manifiesto las transiciones de la vida familiar, que la organización de la misma es el resultado de las tradiciones y las condiciones de vida de la sociedad postmoderna.

A continuación, queremos presentar las relaciones intergeneracionales desde las ambivalencias y contradicciones existentes entre las biografías y las vivencias de los padres, que podemos denominar como «normales y siguiendo las normas y las tradiciones» y las de los hijos e hijas obligados a crearse unas biografías que podemos denominar de «bricolaje» (Beck).

\section{Ambivalencias entre la «biografía normal» de los padres y la «biografía bricolaje» que tienen que crear los hijos e hijas}

Las entrevistas reflejan claramente las contradicciones que se viven en la familia entre la existencia de formas tradicionales de vida (normas, valores, con- 
troles institucionalizados), en las que vivieron la transición a la vida adulta los padres («una biografía normal») y la exigencia de individualización, en la que se ven obligados a vivir sus hijos en la sociedad de la segunda modernidad («biografía bricolaje», una biografía que ellos mismos se van creando, sin que existan unas normas u obligaciones predeterminadas).

Las contradicciones y ambivalencias se acentúan, porque los grandes cambios experimentados, como la tolerancia, la emancipación de la mujer, la liberación de las personas, no tiene continuidad y son regresivas: las nuevas condiciones del mercado de trabajo, el aumento de las desigualdades sociales, la libertad individual continúan dependiendo mucho del nacimiento y del origen social de los padres. Para una parte de la sociedad, es difícil asegurarse una existencia a través del mercado, esto es, fuera de la familia, y la obtención de seguridad va unida a obtener una posición estable en el mercado de trabajo, que cada día es más inalcanzable. Los padres dudan de si los esfuerzos realizados para vivir y acomodarse a la nueva sociedad eran necesarios y, al final, sueñan con que a sus hijos e hijas les iría mejor si se comportaran como ellos y ellas lo hicieron en su juventud.

En este trasfondo, los hijos están obligados a construir su propia biografía, sin depender de la estabilidad de los contextos o de las tradiciones entre las que se mueven, pero no disponen de los medios necesarios para llegar a las últimas consecuencias y se ven abocados a compartir, durante más tiempo del que ellos quisieran, la vida, más bien parte de ésta, con sus padres, y "poder disfrutar de la solidaridad familiar». El alargamiento de la estancia en el hogar paterno se convierte en una "obligada vida en familia». Esta situación viene como obligada, porque los hijos, a pesar de todo, saben que en la sociedad en la que viven les ha convertido en actores fuera y dentro de la familia y que son ellos los que tienen que asegurarse, a través del mercado, cuya evolución desconocen, su existencia y, planificar su biografía.

Vivir solo exige disponer de unas seguridades sociales, iniciar nuevas formas de relación, nuevas redes de solidaridad, adquirir libremente nuevas obligaciones, experimentar otras formas de intimidad, y esto también tiene sus riesgos.

En las entrevistas realizadas, predominan las biografías de los padres y madres que han vivido la familia, las relaciones de género y la sexualidad bajo el dominio del patriarcado: el matrimonio significaba "casi» la única posibilidad de abandonar la familia de origen, de tener relaciones sexuales, de tener hijos, de formar parte de una familia.

A (padre): Es que antes no podías cogerle nada a la mujer si no te casabas, lo primero que te exigía ella: si nos vamos a casar, sí; si no, no. Aquí no toques. J (madre): Tampoco, no te pongas en ese plan..., que tampoco es eso.

A (padre): Y hoy en día, vas tu con tu novia, alquilas un apartamento y te vas a dormir con ella, pero anda, hazlo antes.

$\mathrm{J}$ (madre): Antes era más rígido. 
M (madre): Si yo con éste [dice la madre refiriéndose a su marido] hemos estado 7 años y medio de novios y casi no hemos salido tarde, alguna vez si eran fiestas, pero a mí me hacían ir más pronto a casa hasta que me casé. Ahora se van con las novias por ahí y con los novios, que es lo que funciona. Nosotros no nos hemos ido en la vida, ni un mínimo viaje... Pues fíjate como lo vemos, totalmente distinto.

(Entr. 07.txt - 7:40 (451:461))

Los padres no tenían fácil conseguir una cierta intimidad en sus relaciones de pareja y "ahora son otros tiempos», pero «tienen confianza en los dos» $y$ que no surja un embarazo no deseado en "este momento de su vida».

A (madre): Aquí viene Jesús [novio de una hija], algunas veces dice buenas, están un ratito con nosotros y luego se van a la habitación de María. Yo entiendo que necesitan su espacio y sus momentos de intimidad.

J (padre): A mí no me dejaban hacer eso en tu casa. Estábamos en la salita delante de todo el mundo.

A (madre): Eran otros tiempos. Y como a nosotros nos fastidiaba un poco ese tema, porque si queríamos hablar de algo, en ese momento no podíamos, quizás los comprendamos más a ellos. Y también tienes confianza. El mundo cambia.

J (padre): Eso sí, yo tengo confianza en los dos, son mayores y están enterados. Y ellos saben la circunstancia y lo que les perjudicaría el tener algún desliz en este momento de su vida. También tienen mucha información.

(Entr. 04.txt - 4:46 (629:647))

Antes, las relaciones sexuales estaban permitidas si pensaban fundar una familia y estaban casados, mientras ahora creen que sus hijos no buscan algo definitivo, que tienen relaciones de pareja momentáneas y que pueden no ser definitivas.

L (madre): No piensan en lo que pensábamos nosotros. Nosotros pensábamos en fundar una casa, tener hijos, comprar un piso, y no en irnos por ahí, sino trabajar pa eso.

(Entr. 06.txt - 6:9 (155:157))

En el caso de la mujer, la transición a la vida adulta se realizaba a través del matrimonio, incluso en el caso de las mujeres que se veían obligadas a abandonar pronto la escuela, se ponían a trabajar en casa — ayudando a la madre en las tareas del hogar - o fuera de su casa — entregando todo lo que ganaban a sus padres - . Al casarse, muchas de ellas pasaban de la dependencia paterna a la dependencia de su marido.

El modelo tradicional en el que los padres vivieron la transición al trabajo, a la vida adulta, a la vida independiente, estaba regulado, y no dependía tanto del dinero que hubieran ahorrado, del empleo, de las seguridades que se tuvieran, sino, y sobre todo para las mujeres, de si se tenía o no una pareja estable. 
La sexualidad, independizarse, sólo estaba permitido en el marco de una relación estable y socialmente permitida: el matrimonio.

Noviazgo, ahorrar, empezar a pagar la casa, casarse, era como los estadios para alcanzar la independencia. Transición a la vida adulta y abandonar la familia de origen iban unidas al matrimonio.

$S$ (padre): Era otra forma de vida, pero poco más o menos era lo mismo, se daban las mismas circunstancias, igual en mis tiempos nos hacíamos adultos antes, porque, al casarnos, pasábamos a depender de nosotros antes que hoy. Hoy los jóvenes están más tiempo en casa, en mis tiempos, nos emancipábamos mucho más pronto. Yo con la edad de mi hijo ya estaba casado. Nos casábamos, nos íbamos de casa y teníamos otro tipo de problemas, ya no dependíamos de nuestros padres para solucionar las cosas. Es que hoy la gente joven está viviendo en casa a los 30 años, antes era raro una persona que con 30 años siguiera viviendo con sus padres, sobre todo el hombre.

(Entr. 38.txt - 15:61 (667:675))

Reconocen y aceptan que en la sociedad postmoderna han perdido valor las tradiciones que orientaban sus biografías, pero están dispuestos a aceptar las condiciones que indirectamente imponen sus hijos para que éstos no abandonen la casa sin unas ciertas seguridades: pareja, trabajo y vivienda. Su preocupación no es que retrasen la salida de casa, sino que no estén preparando la salida como ellos lo hicieron: con una relación de pareja y planificando con ella la salida de casa de los padres, aunque reconocen que las condiciones estructurales no lo permiten.

M (madre): Pues fíjate cómo lo vemos, totalmente distinto. Pero vemos que como ha cambiado tanto la vida, lo aceptamos. Es que sino, aquí no habría ni un hijo ya, porque si dijéramos, por esto no paso y ellos lo iban a hacer, ¿dónde estarían los 4? Fuera de casa, porque se habrían ido por no aceptarlo nosotros, ¿entiendes? Tenemos que aceptar que ha cambiado.

(Entr. 07.txt - 7:40 (451:461))

$\mathrm{V}$ (padre): Si le planteas a un joven que hoy se tiene que casar o vivir con una chica, que tiene que utilizar el $70 \%$ de lo que gana para poder comprarse una vivienda, estamos hablando de un caso muy concreto: es el joven el que dice «no me puedo casar, entre otras cosas si tengo que dedicar un $70 \%$ de mi sueldo a la vivienda y tengo un contrato de dos años de trabajo y el piso lo tengo que financiar a 18 años, los otros 16 ¿cómo lo pago?, porque no tengo nada, ya que dentro de dos años no sé lo que estaré haciendo», ése es un problema añadido.

(Entr. 30.txt - 12:12 (144:147))

$\mathrm{M}$ (madre): Ni que digan: «Oye, pues yo me quiero ir ya porque estoy amargado...». Porque para eso le diría: «Mira, ya tenéis vuestros sueldos, coger la 
puerta e iros...». Mi marido igual... Quiero decir que el día que se vayan será porque ya se quieren ir, porque lo están deseando, porque es lógico. También nosotros lo pensamos que es lógico, que se vayan. Estarán deseando hacer su vida. Muchas veces tienen que depender de ahora vengo, ahora entro, ahora salgo..., y porque hacen lo que quieren. Vienen cuando quieren, entran cuando quieren y salen cuando quieren, todos... Ya sabes tú como está. Yo no sé tú lo qué harás, igual eres formalica, pero si llegas un día a las 5 de la mañana, no creo que a tu madre le pidas perdón ni a tu padre excusas. Si te quieres ir un fin de semana, ¿a que te vas? Pues estos hacen igual. Si se van, no preguntan "¿me puedo ir?», no, «me marcho, vendré tal día, ya he llegado, ya estoy...». Eso es lo que hacen nuestros hijos, me imagino que será lo normal ¿no? Lo que hoy en día hacen todos.

(Entr. 07.txt - 7:33 (429:443))

El concepto de «ambivalencias» o «contradicciones» resumiría la situación actual: los padres han vivido la familia como una institución cuyas normas les obligaba a tener que comportarse de una forma determinada, les privaba de libertades, y al mismo tiempo les ofrecía solidaridad y los sancionaba con pri-

\section{Contradicciones y \\ ambivalencias}

\section{Perspectivas de los padres}

sobre la biografía propia y la de los hijos

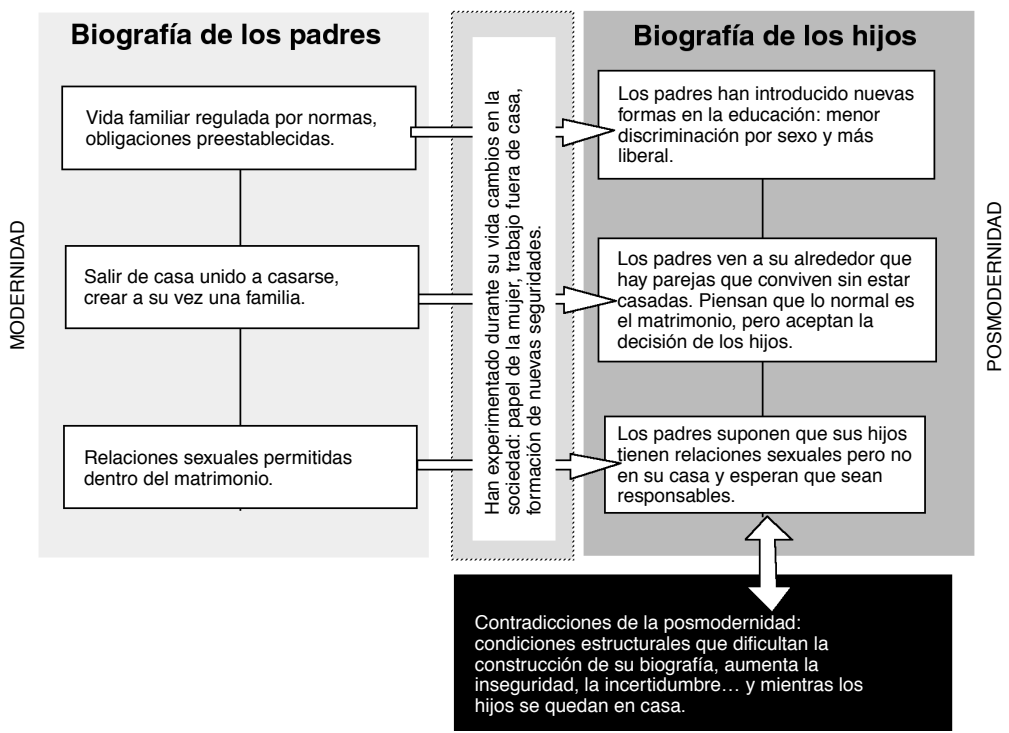

Gráfico 1. 
vación de la misma si alguien se saltaba las normas; para ellos, crear su propia familia era la única posibilidad de obtener ciertas libertades y acceder a la vida adulta. Ellos mismos, durante su vida adulta, han vivido los grandes cambios que se han dado en la postmodernidad: la exigencia de la mujer que quiere vivir su propia vida — formación, trabajo, independencia económica - y la posibilidad de obtener seguridad o seguridades fuera de la familia, por nombrar solamente dos grandes cambios. Con grandes esfuerzos y con mayor o menor "éxito", se han ido acomodando a la sociedad actual, han apostado por una mejor educación para sus hijos, han intentado no discriminarlos por razón de sexo, se han ido acomodando a las exigencias de libertad y, al mismo tiempo, les han ofrecido la familia como lugar de solidaridad y apoyo psicológico.

En el gráfico 1, queremos resumir las perspectivas que los padres y las madres tienen sobre su propia biografía y sobre sus hijos e hijas.

\section{La creación de un hogar dentro del hogar de los padres: una forma de "salir de casa de los padres"}

El alargamiento de la fase de la transición de los hijos a la edad adulta genera nuevos retos al sistema familiar. Retos que atañen de forma directa al equilibrio de la familia y que, por tanto, requieren de ésta una adaptación a la nueva situación, que supone el incremento temporal de la estancia de los hijos en casa. Se observa como las diferentes familias han ido adaptándose a la experimentación de esta nueva situación, teniendo como denominador común el objetivo prioritario de evitar conflictos que las desestabilicen y que las puedan conducir a un clima de malestar y de conflicto permanente.

Esta adaptación parece haber requerido que se efectuaran determinados cambios en el seno familiar, cambios que afectan a todos sus miembros. Entre esos cambios, se observa, en las narraciones biográficas de los jóvenes, un cambio de actitudes en la convivencia familiar, tanto por parte de los hijos como por parte de los padres. Estos cambios de actitudes se constatan sobre todo en la adaptación de las normas y las reglas de convivencia a los momentos biográficos de los jóvenes y las jóvenes.

Los jóvenes, en su crecimiento biológico (edad) y social, van teniendo unas determinadas necesidades, de libertad, de independencia, de autonomía en las decisiones, de madurez, que van siendo cubiertas en mayor o menor grado por las diferentes familias a las que pertenecen, y que, dependiendo del grado de su cobertura, han generado o no la experimentación de un mayor número de conflictos y de crisis dentro de las familias. En el resultado general, tras analizar el conjunto de familias en las que viven los jóvenes entrevistados, parece observarse una cierta actitud de flexibilización progresiva por parte de la autoridad paterna y materna en cuanto a la formulación y el cumplimiento de determinadas normas y reglas a cumplir por parte de los jóvenes dentro del hogar familiar.

Se observa que los patrones de comunicación entre padres e hijos van cambiando y adaptándose a las nuevas circunstancias que plantea el cumplimien- 
to de determinadas fases biológicas y psicosociales del desarrollo de la transición. Este desarrollo adaptativo finaliza con situaciones de convivencia familiar en las que el joven y la joven disponen de total libertad para entrar y salir de casa, para hacer lo que quieran fuera de la misma, para tomar sus propias decisiones, de tal forma que se puede observar, como en muchas ocasiones se da, una emancipación encubierta o una emancipación dependiente.

También se observa un cambio en los jóvenes referido a la manera de observar la convivencia dentro del hogar familiar. Es una actitud más madura, más comprensiva de las actitudes de los padres y donde el diálogo y la comunicación son los aspectos que presiden las actitudes dentro del hogar familiar. Estas actitudes son destacadas sobre todo por los jóvenes que tienen una edad más madura y que siguen conviviendo en el hogar familiar.

Se deduce de los relatos de los jóvenes que se intercambian actitudes basadas en la relación entre padres e hijos. En los primeros momentos del proceso de transición de los jóvenes (edades más tempranas), se produce una relación vertical, mientras que posteriormente se pasa por una orientación más horizontal y de igualdad, debido al alargamiento del proceso de transición.

Merxe, 27 años: Buena. Yo estoy muy bien aquí, estoy muy bien con mis padres, también es por mi carácter, yo no he sido chica rebelde, no es que yo acepte o acate todo, pero estoy muy bien, tenemos una relación de diálogo continuo y bien, no tengo ningún problema, no me siento presionada, y hago lo que quiero dentro de unos límites, pero te vuelvo a repetir, que estoy muy bien. (Entr. 13Y.txt (104:108))

Sara, 25 años: Y la relacion familiar es muy buena. Se puede resumir así. Podemos tener maneras distintas de ver las cosas, pero cada persona tiene su opinión y tampoco hay que ser tajante ni cerrarse en banda en una cosa. (Entr. 19Y.txt (175:183))

Javier, 26 años: No hay conflicto y hay comunicación. Pensamos diferente en unas cosas e igual en otras. Mejor es ahora que hace unos años, cuando había más roce y más conflicto de intereses. (Entr. 36Y (400:402))

Se observa como, si este movimiento adaptador no se produce, se generan y se viven determinados conflictos o se mantienen larvados algunos que se detectan en la convivencia cotidiana de los jóvenes y las jóvenes dentro del hogar familiar. La experiencia de vivir en casa puede conllevar y conlleva la aparición de pequeños conflictos familiares que se experimentan en todas las familias. La cena, la televisión y el compartir el baño son escenas cotidianas en las que los jóvenes y las jóvenes conviven con sus padres. Se observa que, conforme los hijos tienen un mayor grado de independencia, pasan gran parte del día fuera del hogar familiar, se reduce notablemente el nivel de conflictos.

Podríamos afirmar que esta fase puede tratarse como la anterior a la independencia final, en la que los hijos van pensando en una salida del hogar familiar, van planificando inconscientemente la misma y, al mismo tiempo y de forma sincronizada, van cambiando los patrones de relación familiar que hasta 
entonces mantenían. Este proceso es más bien una adaptación a la nueva situación de la prolongación de la estancia del joven en el hogar familiar.

Ahora bien, la gestión del espacio interior, de la intimidad del hogar familiar, es un aspecto en el que no se puede observar una tendencia general mayoritaria y, en ocasiones, es motivo de discusión o de conflicto entre padres e hijos. El hogar familiar, como espacio de convivencia en el que uno pueda tener la posibilidad de invitar y de compartir con personas externas a la familia, es objeto de diferentes planteamientos familiares.

Son tres formas de actuación las que se observan en el seno de las familias de los jóvenes entrevistados: la libertad total de los miembros de la familia a la hora de invitar amigos o compañeros a casa, la posibilidad de llevar amigos o compañeros a casa siempre y cuando los padres conozcan este hecho y la determinación del espacio familiar como espacio vetado a las amistades de los hijos.

En la elección de estas posibilidades, parece determinante, a los ojos de los jóvenes, el grado de confianza que los padres depositan en los hijos y en el grado de responsabilidad que les conceden. Muchos padres parecen mostrar cierto agrado o ser favorables a que los hijos traigan a sus amigos a casa, para conocerlos, quizás motivados por el control de las amistades de sus hijos. Quizás la experiencia más extendida es la de la invitación condicionada, y la menos frecuente, la de la libertad total.

Antonia, 26 años: No, yo tengo toda la libertad, yo tengo plena libertad de... consultándolo antes. (Entr. 33Y.txt (245:246))

Carlos, 24 años: No sé! igual a la hora de invitar a alguien a casa. Tú invitas y... ¡hombre!, dices: «va a venir no sé quién, fulanito de tal», pero lo tienes que anticipar y ya igual se organizan y lo entienden, pero estás igual en una reunión por ahí: «oye veniros a casa», ;no!, siempre le choca un poco que traigas a gente de fuera o no sé. (Entr. 31Y (234:238))

Joaquín, 25 años: No, nunca, esta casa es grande. Si estaban ellos, pues estábamos en mi habitación, si no estaban, pues en el salón o la cocina, pero nunca me han dicho nada, además, siempre dejábamos todo bien, si comíamos algo, los metíamos en el lavavajillas y ya, tirábamos las colillas a la basura, vamos, mis viejos ya sabían lo que hacíamos, pero no me decían nada, mientras no montara una macrofiesta en casa o algo así. (Entr. 16Y.txt (458:463))

Rosa, 24 años: Mis padres nunca me han dicho nada, creo que aparte porque no he abusado de eso, en el sentido de que si yo he traído a alguien a cenar a casa, mis padres siempre lo han sabido. Con las parejas que he salido, mis padres les han conocido a todos, o incluso amistades. Es más, mi padre me decía: "¿por qué no traes a tus amigos aquí?». De mi cuadrilla, las conocen a todas. Siempre, y todas las cuadrillas con las que he salido aquí, en B..., todas conocen a mis padres. Es un interés que tampoco lo hacían en el plan de vigilarme, de controlarme, sino porque ha surgido así. (Entr. 22Y (270:278))

Anabel, 26 años: Me ponían normas, ya que mi casa es sagrada. Para mis padres, es su sitio, su espacio, y no quieren a nadie del exterior. Tenía intimidad en 
mi habitación, pero muy poca. Me arreglaba muy mal porque no paraba en casa en ningún momento. (Entr. 38Y (298:301))

Ignacio, 23 años: Pues que veníamos a casa y cuando nos íbamos no dejábamos nada por medio, ni eso, pero la madre se empezó a mosquear porque igual fumábamos y quemábamos algo, pitillos, ceniza y claro empezaba a chillar "iQue no traigas a amigos a casa!», y para estar así, pues dejé de traer amigos a casa. No quiero broncas. Ahora, si venimos, nos metemos en la cocina, cenamos y nos vamos. (Entr. 23Y.txt (533:538))

Chema, 27 años: No me encuentro libre. Yo no traigo a nadie a mi casa. Yo me voy fuera... No puedo invitar a un amigo si fuma, ya que en mi casa, en mi casa, no se permite fumar en el interior de la casa... (Entr. txt32Y.txt (635.637))

Hay claras evidencias de que existen conflictos ocultos, larvados, que permanecen abiertos, y que no salen a la luz, referidos principalmente al no cumplimiento de las expectativas paternas en determinadas áreas, como son los estudios o el trabajo. Sobre todo, este aspecto de "conflictos ocultos» es especialmente observable en el caso de los jóvenes que se han emancipado del hogar familiar, que viven de forma independiente.

Ainoa, 25 años: Los padres siempre tienen problemas con los hijos cuando queremos ir soltándonos de la familia. A ellos no les gustaba mi manera de querer vivir. Tener una cría soltera pues ya veas como se ponían. Ahora aceptan o callan, pero no hay problemas. (Entr. 41Y (334:338))

Anabel, 26 años: Ahora, en estos momentos, es normal, ni buena ni mala. Se puede decir que nos llevamos bien, pero tampoco demasiado bien, y cuando vivía en casa, bastante mal. (Entr. 38Y (276:278))

Igualmente, se observa la existencia de que se producen escenificaciones claras de evitar conflictos, cuya máxima expresión es la de aquellos jóvenes que evitan pasar mucho tiempo en casa con sus padres. La no presencia en casa es todo un síntoma, al mismo tiempo que una estrategia que siguen aquellos hijos que quieren evitar la aparición de conflictos. De tal forma que si el nivel de conflictividad es alto dentro del hogar familiar, los jóvenes reducen considerablemente el tiempo de estancia en el hogar familiar, y lo dedican a otros espacios y ambientes, como puede ser el grupo de iguales.

Estos jóvenes viven en el mismo hogar familiar que sus padres, pero no conviven la mayor parte del tiempo con ellos. Digamos que no comparten tiempo con la familia, o lo reducen a límites mínimos. El grado y el nivel de transacciones entre padres e hijos decae y proyectan sus transacciones positivas en otros espacios en los que encuentran una mejor respuesta o un menor grado de conflictividad; peer groups, los amigos, son sus principales lugares de convivencia y de realización personal, de tal forma que las interacciones entre los miembros del hogar decaen en momentos «clave» dentro del horario familiar, y es precisamente en esos espacios donde «emergen» los roces y conflictos familiares. 
Chema, 27 años: No cesaron los conflictos y todavía perduran. Siempre están relacionados con que yo no me adapto a sus maneras de vivir. Su orden, su limpieza, sus horarios, su música, etc. yo no los puedo aceptar, y en eso chocamos. No me adapto a tenerlos presentes, verlos, cruzarme en el pasillo, en el comedor, con ellos. A pesar de ello, creo que ninguno somos felices cuando estamos mucho tiempo juntos. Creo que nos esforzamos en «vivir y dejar vivir a los demás», pero no siempre se consigue. (Entr. 32Y.txt (613:619))

Entrevistador: ¿Y en casa, podéis hablar de cosas importantes con tus padres? Ignacio, 23 años: Es que no estoy nunca en casa.

Entrevistador: Bueno, pero los raticos que estás...

Ignacio, 23 años: Bueno, con la vieja más, pero siempre acaba todo en discusión, entonces prefiero callarme, irme y ya está, dejarle a la madre chillando. (Entr. 23Y.txt (392:399))

Germán, 26 años: Duermo y aparte de estas horas de la tarde, sí suelo estar de cuatro a seis, pero esas dos o tres horas de ahora y dormir, prácticamente no estoy nada más en casa, así que no discuto. (Entr. 10Y.txt (373:375)

Josetxo, 23 años: Es que no tengo relación. No estoy nunca en casa. No sé, con la madre a veces hablo algo más, pero muchas veces empezamos hablando y acabamos discutiendo, pues le empiezo a contar el fin de semana y que nos hemos ido por ahí y hemos hecho esto y tal y siempre saca el tema del dinero o que estoy todo el día por ahí, y para rematar, si me dice "más te vale que aproveches para sacarte el carnet», entonces ya, ya, me voy y que le den. (Entr. 9Y.txt (547:556))

\section{Interpretaciones}

La solución a largo plazo más aceptable y estable es renegociar el contrato de la familia heterosexual. Ello incluye compartir las tareas domésticas, la participación económica, la participación sexual y, sobre todo, compartir plenamente la paternidad. Esta última condición es crucial para los hombres, porque sólo en esas circunstancias puede alterarse el efecto "Chodorow» y las mujeres podrían reproducirse no sólo como madres, sino como mujeres que desean a los hombres, y los hombres podrían criarse no sólo como amantes de las mujeres, sino como padres de los hijos. (Castells, 1998, p. 261)

1. En el análisis presentado, no pretendemos definir si las transiciones de los jóvenes vienen marcadas por una armonía de conveniencia o si podemos hablar de semiindependencia, o introducir el concepto de autonomía personal, sino si se dan las condiciones para una redefinición de la familia, las relaciones de género, la sexualidad.

Las biografías de los padres y las madres entrevistados han vivido la familia, las relaciones de género y la sexualidad bajo el dominio del patriarcado, que permanece y sobrevive bajo la protección de estados autoritarios y fundamentalismos autoritarios: el matrimonio significaba «casi» la única posibilidad de abandonar la familia de origen, de tener relaciones sexuales, de tener hijos, 
de formar parte de una familia. Las mujeres se «debían» realizar como madres, se ocupaban de la organización de la familia, de todas las tareas del hogar y, en caso de conflicto y por la paz familiar, «debían ceder», mientras que los hombres no participaban en las tareas internas de la familia. En las entrevistas, predominan estas biografías, junto a otras en las que las mujeres también quieren realizarse con su personalidad, con su presencia en la sociedad, con su trabajo fuera de casa, y algunos hombres que también participan en las tareas de la familia, y están dispuestos a perder sus privilegios y «a trabajar junto a las mujeres para encontrar nuevas formas de amar, compartir la vida y tener hijos» (Castells, 1998, vol. III, p. 383) ${ }^{2}$.

Los jóvenes, por su parte, viven la necesidad de construir su propia biografía sin depender de la estabilidad de los contextos o de las tradiciones entre las que se mueven. Se ven obligados a tomar decisiones individuales - en materias relacionadas con la educación o el empleo, en sus relaciones personales, pero también con el ocio- que pueden ser de gran influencia para su vida futura, sin ser capaces de prever claramente todas las implicaciones de sus opciones. Los jóvenes deben tomar las decisiones que conllevan el riesgo de equivocarse. Están obligados, si quieren sobrevivir en la sociedad postmoderna, de la segunda modernidad, a construir, formar una personalidad flexible, capaz de llevar a cabo continuamente su propia identidad, buscar y dar respuesta a las nuevas situaciones. En la sociedad actual, de la segunda modernidad, ya no vale acudir a la tradición para definir su personalidad, ni acomodarse a los roles sociales, lo que vale es la experiencia real que las personas tienen en sus relaciones.

Al mismo tiempo, también hemos constatado que, en la sociedad actual, caracterizada por la individualización, la desestructuración de la sociedad civil, la flexibilidad del mercado laboral, la privatización de los servicios y la paulatina desaparición del Estado de bienestar, las familias ofrecen a sus miembros una seguridad material y un apoyo psicológico. Las familias se convierten en «una red de apoyo».

2. Creemos que se da, de hecho, una ruptura entre lo que los padres y las madres viven, consideran valioso, creen que sería bueno para sus hijos, y lo que los hijos realmente hacen, piensan y valoran. La cuestión no tendría demasiada trascendencia y sería considerada como una cuestión menor, pues los jóvenes siempre han contestado el mundo de los padres y de los adultos (principio de emancipación).

La gravedad de la ruptura viene dada por el hecho de que las instancias socializadoras tradicionales que antes suplían a los padres, tales como la escuela, el mercado de trabajo y los grupos más o menos organizados en asociacio-

2. En la investigación, que sirve de base a este artículo, hemos tenido dificultades para incluir las formas de familias que se constituyen con una madre sola y sus hijos, o parejas de hecho o parejas homosexuales con hijos, las cuales, en muchos casos, están creando nuevas redes que ofrecen un apoyo emocional y material. 
nes de tiempo libre, y acompañaban a los hijos en su independencia de los padres hacia la integración social, laboral y en la vida adulta, hasta crear una nueva familia como signo externo de independencia pero también de haber alcanzado la madurez emocional y en definitiva la edad de adulto, responsable y autónoma, han dejado de servir de referencia.

Estas instituciones ya no suplen a nadie, ni tienen la confianza de los padres y las madres, y están muy lejos de asumir la responsabilidad de acompañar a los jóvenes hacia ningún sitio. Como señalaba Brater (1997, p. 149 y s.), la individualización de la sociedad como consecuencia de la libertad y de la democracia supone que las personas y las organizaciones optan por valores y concepciones múltiples sin un criterio discriminador, todo es posible, la fuerza socializadora pierde prestancia. En su lugar, adquieren especial relevancia los peer groups, que a su vez se apartan del control de los padres. Éstos no saben lo que ocurre en esos espacios y sí saben que para los hijos se ha convertido en un espacio privilegiado y preferido incluso a la propia familia.

3. Hemos intentado mostrar las contradicciones en las que se encuentran los padres y las madres. Ellos mismos han hecho un esfuerzo ímprobo para conseguir el estatus social del que ahora disfrutan y que se ha basado en la creencia en el progreso como elemento justificador de los esfuerzos: estudios, formación, búsqueda de trabajo, experiencia laboral desde muy jóvenes, independencia sin desvinculación de sus progenitores, todo para conseguir un piso, un modo de vida con cierto desahogo económico, puesto que consideran muy alto el valor del trabajo. La mujer se integra en el mercado laboral como un signo de liberación, de independencia e individualización, al mismo tiempo que los padres mantienen la mentalidad de la familia como un lugar de trueque, de refugio emocional, de trampolín para mejor vida para los hijos. En una palabra, han construido condiciones de vida correspondientes a la sociedad postmoderna, mientras que, en parte y en lo referente a los hijos, desean que se comporten como ellos lo hicieron en la familia industrial. Les gustaría que sus hijos se comportaran como ellos se comportaron, algo imposible, porque para los hijos no se dan las condiciones que se dieron para los padres. Junto a los fenómenos de transferencia y proyección de carácter psicológico por parte de los progenitores, se da un fenómeno social o sociológico, la negación de las nuevas condiciones de vida en las que crecen los hijos en la sociedad actual: un alto grado de libertad, las necesidades básicas y algunas más cubiertas, grandes posibilidades de ocio y tiempo libre, dinero para gastar sin necesidad de ganarlo, pocas expectativas de trabajo seguro y bien remunerado. El valor del trabajo como sentido de vida deja paso a otros como el vivir bien al día, trabajando o sin trabajar, el disfrutar del momento.

Es decir, los padres se encuentran en una situación de grandes contradicciones. Ellos mismos viven o, mejor, han alcanzado la postmodernidad. El ideal para sus hijos lo mantienen en la modernidad, en la era industrial, en una socialización lineal que ya no se da o sólo tiene lugar en algunos casos. 
4. Estas contradicciones son reflejo de las que existen en la propia sociedad (Beck, Beck-Gernsheim, 1993; Beck-Gernsheim, 2000), que, al mismo tiempo que preconiza un ideal postmoderno (ser uno mismo), no pone los medios para que cada uno pueda alcanzar este ideal, ya que tenemos que buscar soluciones biográficas estando enfrentados a contradicciones sistémicas.

A la generación adulta, la de los padres, se les exige, en el ideario postmoderno, trabajar fuera del hogar, disponibilidad laboral. La flexibilidad se entiende a favor de la empresa, y se traduce en disponibilidad de horario, de puesto de trabajo y de lugar. Con ello queda poco espacio para la vida familiar. Sin embargo, la sociedad no libera a la familia de algunas obligaciones, como son las del sustento de los jóvenes, la financiación de sus necesidades y la integración laboral. La familia se convierte en el hotel en el que la mujer asume con frecuencia la posición del hotelero al servicio de los miembros del hogar, que con gusto hacen uso de los servicios: lavado de ropa, preparación culinaria, limpieza, coordinación de horarios y la correspondiente paga de fin de semana.

Como dice Beck-Gernsheim (2000, p. 109), el contrato generacional pasa por el contrato de género, pero éste también pasa por un nuevo contrato social, que regule las nuevas funciones de la familia y su lugar en una sociedad postmoderna, en la que la conciliación de la vida familiar y la laboral resulte factible, donde la maternidad y la tenencia de hijos no suponga un riesgo adicional. La vida postmoderna convertida en proyecto deja de existir para los más jóvenes, lo que crea una paradoja o una contradicción: abocados a la planificación, los jóvenes no pueden planificar su futuro, porque las condiciones laborales no se lo permiten, la vida se ha hecho demasiado compleja y cada uno se las debe ingeniar como pueda. "Así pues, la planificación reflexiva de la vida se vuelve imposible, excepto para la élite que habita el espacio atemporal de los flujos de las redes globales y sus localidades subordinadas» (Castells, 1998, tomo II, p. 33).

5. El entendimiento entre los diversos miembros del hogar y la toma de decisiones en las familias se hace difícil, dado que cada uno tiene sus gustos, sus preferencias y el mundo externo está lleno de ofertas. Ante las dificultades, se opta por dialogar formalmente algunos aspectos esenciales de la vida familiar, pero todo lo demás se queda ahí, como si se hubiera negociado. Bajo el manto de la tolerancia y el diálogo, se esconden una vida de relaciones sin relaciones, las contradicciones se hacen patentes, pero no se explicitan.

6. La familia va acomodándose a los cambios económicos, demográficos, culturales, aumenta la disponibilidad para que los jóvenes permanezcan en casa. La familia se ha convertido en una familia de la negociación continua. La negociación se inicia para evitar conflictos, sin embargo, la aparente armonía, una armonía forzada, se convierte "en un entendimiento distante» en un "compromiso fruto de las negociaciones entre generaciones». De este modo, ambas partes pueden guardar las formas y llevar cada uno su camino: ignorancia refle- 
xiva en el mejor de los casos «indiferencia calculada», una especie de alto el fuego entre yoes encaramados... "La mutua aceptación del "espacio propio", de la "vida propia", corresponde a una economía de conflicto: dejar hacer a cada uno lo que quiera es, finalmente, más barato y efectivo» (Beck, 1997, p. 215).

7. La mayoría de las familias continúan teniendo como función principal la de ser refugio de intimidad y la de proporcionar toda una serie de servicios no monetarizados (Esping-Andersen, 2000, p. 49-67) que sustituyen la inexistencia de fuentes de bienestar para los jóvenes. Sin ingresos propios, los jóvenes no tienen posibilidades de llevar una vida autónoma fuera del hogar de origen. La familia se siente obligada a continuar con sus funciones asistenciales, dadas las carencias del Estado de bienestar y la imposibilidad, por falta de medios, de acceder al mercado para recibir los servicios que, gratis, reciben de su familia. La familia en España, en estos momentos, está absorbiendo una gran parte de los riesgos sociales a los que tienen que enfrentarse los jóvenes. La falta de estructuras de apoyo externas a los hogares origina que aumenten las desigualdades sociales.

8. Nos encontramos en una fase de transición familiar en la que, como dice Castells (1998, vol. II, p. 268), en estas condiciones históricas, las familias y los modos de organizar la vida se están redefiniendo en términos confusos, y más tarde concluye este autor: «La liberación de la familia enfrenta al yo con su propia opresión infligida. La huida a la libertad en la sociedad red abierta llevará a la ansiedad individual y a la violencia social, hasta que se encuentre nuevas formas de coexistencia y responsabilidad compartida que unan a las mujeres, los hombres y los niños en una familia igualitaria reconstruida, en la que puedan convivir mujeres libres, niños informados y hombres inseguros» (ibídem, p. 268).

\section{Bibliografía}

BAUMAN, Z. (1996). «Modernidad y ambivalencia». En BeriaIn, J. Consecuencias perversas de la modernidad. Barcelona: Anthropos, p. 73 y s.

- (2004). «Flüchtige Moderne». Conferencia pronunciada en el Congreso de ANSE sobre Die Werteproblematik als Herausforderung für Praxis und Konzept von Supervision und Coaching (7 de mayo de 2004, Leiden /NL).

BECK, U. (1986). Risikogesellschaft. Frankfurt a. M.: Suhrkamp.

- (1997). «Demokratisierung der Familie». En BECK, U. (ed.). Kinder der Freiheit. Frankfurt a. M.: Suhrkamp, p. 195-216.

- (ed.) (1997). Kinder der Freiheit. Frankfurt a. M.: Suhrkamp.

BeCK-GERnSHEIM, E. (2000): Was kommt nach der familie? Einblicke in neue Lebensformen. München, C.H.: Beck Verlag.

BeCK, U.; BeCK-GERnSHeIM, E. (1993). «Nicht Autonomie, sondern Bastelbiographie. Anmerkungen zur Individualisierungdikussion am Beispiel des Aufsatzes von Günter Burkart», en: Zeitschrift für Soziologie. Heft 22, junio. 
Biggart, A.; Walther, A. et al. FATE (2004). Families and transitions in Europe: Comparative report. Coleraine: University of Ulster, Draft, oct. 2004.

Brater, M. (1997). «Schule und Ausbildung im Zeichen der Individualisierung». En BECK, U. (ed.). Kinder der Freiheit. Frankfurt a.M.: Suhrkamp, p. 149-174.

CASTElls, M. (1998): La era de la información. Vol. 2: El poder de la identidad. Vol. 3 : Fin de milenio. Madrid: Alianza Editorial.

Du Bois-Reymond, M.; López Blasco, A. (2003). "Yoyo Transitions and Misleading Trajectories. From Linear to Risk Biographies of Young Adults». En LópEZ Blasco, A.; McNeish, W.; Walther, A. (eds.). Dilemmas of Inclusion: Young People and Policies for Transitions to Work in Europe. Bristol: Policy Press, p. 19-42.

ESPING-ANDERSEN, G. (1999). "Trabajo, familia y estado de bienestar». En Castells, M.; Esping-Andersen, G. Transformación del trabajo. Colomers (Barcelona): La Factoría Cultural.

- (2000). Fundamentos sociales de las economías postindustriales. Barcelona: Ariel. FlaQUer, L. (1998). El destino de la familia. Barcelona: Ariel.

Hernández, J.; LÓPEZ Blasco, A. (2001). La familia ante el cambio social. Valencia: Nau Llibres.

- (2005, en prensa). Pluralidad de formas de familia: Las familias monoparentales con hijos a cargo. Valencia: Nau Llibres.

INJUVE (2005). Informe Juventud en España 2004. Madrid: Ministerio de Trabajo y Asuntos Sociales.

López Blasco, A. (2003a). Families and Transitions in Europe. Qualitative Survey. National Report Spain. FATE-EU Project. Coleraine: University of Ulster.

López Blasco, A.; McNeish, W.; Walther, A. (eds.) (2003). Dilemmas of Inclusion: Young People and Policies for Transitions to Work in Europe. Bristol: Policy Press.

Moreno Mínguez, A. (2004). Family and Welfare State in the Southern Countries. The role of family policy. BMFSFJ, European Ministerial Conference, Statement. Strauss, A.; Corbin, J.(1990). Basics of Qualitative Research. Grounded Theory Procedures and Techniques. Newbury Park, Londres, Nueva Delhi: Sage.

Walther, A.; Stauber, B. (eds.) (2004). Final YOYO Report. Tübingen: EGRIS (en prensa).

Andreu López Blasco, doctor en Sociología, licenciado en Sociología por la Universidad de Munich y en Ciencias Políticas y Sociología por la Universidad Complutense de Madrid, investigador numerario en DJI de Munich y profesor asociado en las universidades de Munich y de Valencia. Ha ejercido responsabilidades políticas a nivel local y autonómico en Valencia. Actualmente, es director científico del grupo de investigación AREA y miembro de EGRIS. Es coautor en la obra colectiva Dilemas of inclusion: Young People and Policies for Transition to work in Europe (2003, Bristol). Correo electrónico: andreublasco@terra.es 